\section{Os efeitos do treinamento de força sobre os fatores de risco da síndrome metabólica}

\author{
Effects of resistance training over \\ metabolic syndrome risk factors
}

\section{Resumo}

Introdução: Medidas não-farmacológicas, como a atividade física, vêm sendo recomendadas para prevenção e tratamento de doenças crônicas não transmissíveis. Objetivo: Realizar revisão da literatura para verificar os mecanismos por meio dos quais o treinamento de força provoca alterações metabólicas e celulares, agindo positivamente sobre os fatores de risco da síndrome metabólica. Metododologia: Foram utilizadas as bases de dados Medline, Scielo, Science Direct e Capes. A busca foi restrita aos últimos 10 anos. Os termos utilizados para pesquisa foram: obesity, dislipidemy,hypertension, diabetes mellitus, metabolic syndrome, resistance training, weight lifting, exercise. Resultados: $\mathrm{O}$ treinamento de força atua sobre parâmetros metabólicos e celulares promovendo efeitos positivos no controle e na prevenção dos fatores de risco relacionados à síndrome metabólica, tais como diminuição do peso corporal, aumento da sensibilidade à insulina, aumento da tolerância à glicose, diminuição dos níveis pressóricos de repouso e melhoria do perfil lipídico. Conclusão: A revisão dos artigos científicos apresentados fornece dados que permitem concluir que o treinamento de força pode contribuir de forma efetiva na diminuição dos fatores de risco relacionados à síndrome metabólica.

Palavras-chave: Exercício físico. Síndrome metabólica. Obesidade. Fatores de risco.

\author{
Ana Paula Muniz Guttierres' \\ João Carlos Bouzas Marins ${ }^{2}$ \\ 'Departamento de Nutrição e Saúde do Centro de Ciências Biológicas da \\ Universidade Federal de Viçosa \\ ${ }^{2}$ Departamento de Educação Física do Centro de Ciências Biológicas da \\ Universidade Federal de Viçosa
}


Abstract

Introduction: Non-pharmacological measures, such as practicing physical activity, have been recommended for prevention and treatment of non-transmissible chronic diseases. Objective: To review the mechanisms by which resistance training results in metabolic and cellular alterations that act positively on metabolic syndrome risk factors. Method: The search was limited to the past 10 years, using the Medline, Scielo, Science direct and Capes databases. The terms used in the search were: obesity, dyslipidemia, hypertension, diabetes mellitus, metabolic syndrome, resistance training, weight lifting, and exercise. Results: Resistance training affects metabolic and cellular parameters. It may have positive effects on the control and prevention of metabolic syndrome risk factors, such as reduction of body weight, increase in insulin sensitivity, increase in glucose tolerance, reduction of blood pressure levels at rest and improvement of blood lipid profile. Conclusion: The review gives evidence that resistance training may contribute to reduce metabolic syndrome risk factors.

Key Words: Exercise. Metabolic syndrome. Obesity. Risk factors.

\section{Introdução}

A obesidade, segundo a Organização Mundial de Saúde (OMS) ${ }^{1}$, é considerada um problema de saúde pública que leva a sérias conseqüências sociais, psicológicas e físicas, sendo associada ao maior risco morbimortalidade por enfermidades crônicas não-transmissíveis. A ocorrência de três ou mais morbidades é conceituada como síndrome metabólica, que é caracterizada pelo grupamento de fatores de risco cardiovascular, como hipertensão arterial, resistência insulínica, hiperinsulinemia, intolerância à glicose ou diabetes mellitus tipo 2, obesidade central e dislipidemia $^{2}$. O grupamento da obesidade tóroco-lombar, tolerância à glicose diminuída, hipertrigliceridemia e hipertensão tem sido denominado por certos autores deo "Quarteto Mortal", que condiciona a síndrome plurimetabólica a elevado risco cardiovascular ${ }^{3}$. A síndrome metabólica tem sido descrita como síndrome de resistência à insulina e está evidente que se trata do componente-chave da síndrome metabólica ${ }^{4}$.

A obesidade gera resistência insulínica em nível pós-receptor. Isto provoca hiperinsulinemia compensadora, com sobreestímulo nas células beta do pâncreas, podendo provocar até mesmo falência destas e também insensibilidade dos receptores periféricos. Isto resulta, primeiramente, em tolerância à glicose diminuída, podendo progredir para o diabetes mellitus. A indisponibilidade das células em utilizar a glicose faz com que aumente a liberação de ácidos graxos do tecido adiposo, o que estimula a gliconeogênese hepática, dificultando ainda mais a homeostase da glicose sangüínea. A hiperinsulinemia resultante também reduz a excreção de sódio pelo organismo, o que provoca expansão do volume extracelular, aumentando o trabalho do coração e do sistema cardiovascular periférico. A insulina aumenta a atividade do sistema nervoso simpático, acarretando vasoconstrição, o que aumenta o risco cardiovascular ${ }^{3}$. 
Medidas não-farmacológicas, como a atividade física, vêm sendo aplicadas aos pacientes com síndrome metabólica, visto que o sedentarismo e o baixo nível de atividade física têm sido considerados fatores de risco para a mortalidade prematura tão expressivos quanto o fumo e a hipertensão arterial $^{3}$. O treinamento de força (TF), ou treinamento contra resistência, vem sendo reconhecido como importante componente do programa de condicionamento físico para adultos devido à promoção de diversos benefícios à saúde ${ }^{5}$. Há fortes indícios de que altos níveis de força muscular podem estar associados à diminuição da prevalência de síndrome metabólica ${ }^{4}$.

O Colégio Americano de Medicina do Esporte-ACSM ${ }^{6}$ (1998) preconiza o treinamento de força (TF) ou contra-resistência, ou treinamento resistivo com certas restrições, para pessoas com hipertensão arterial, doença vascular periférica, diabetes mellitus, obesidade ou outras condições comórbidas. No caso da obesidade, defende que o TF é um coadjuvante valioso no treinamento aeróbico. O TF promove aumento da força e resistência muscular localizada, podendo, com isso, melhorar a execução das tarefas da vida diária ${ }^{7,8}$.

Em outro posicionamento, o ACSM $(2002)^{7}$ preconiza o treinamento de força para adultos jovens a partir de uma progressão gradual. A qualidade do programa de treinamento de força deve ser otimizada, seqüenciando a execução de exercícios multiarticulares antes de monoarticulares, de alta intensidade antes daqueles de menor intensidade. Para indivíduos iniciantes, as cargas de treinamento devem corresponder a uma intensidade de 8 -12 repetições máximas (RM). Para indivíduos intermediários e avançados a variação de repetições é maior de 1-12 RM de forma periodizada, sendo enfatizadas 1-6 RM, com descanso médio de 3 minutos com velocidades moderadas de contração (1-2 segundos na fase concêntrica e 1-2 segundos na fase excêntrica da contração muscular). A freqüência de treinamento para iniciantes e intermediários deve ser de 2-3 vezes e para avançados de 4-5 vezes semanais com um dia de descanso no meio da semana.

Os benefícios do TF e a forma de montagem do programa de treinamento para indivíduos com síndrome metabólica estão largamente divulgados, porém a análise das modificações metabólicas e celulares induzidas pelo treinamento contra-resistência que provocam tais benefícios é pouco discutida na literatura. Os dados disponíveis indicam uma associação inversa entre a atividade aeróbica e o condicionamento cardiorespiratório, com a prevalência de síndrome metabólica ${ }^{4}$. Contudo, pouco se sabe sobre a relação entre força muscular e síndrome metabólica. Assim, o objetivo desta revisão de literatura foi verificar os mecanismos por meio dos quais o treinamento de força provoca alterações metabólicas e celulares que podem agir positivamente sobre os fatores de risco da síndrome metabólica.

\section{Metodologia}

Foram revisados periódicos da base de dados Medline e Science Direct. A busca foi restrita aos últimos 10 anos (1995-2005). A inclusão de alguns artigos mais antigos foi necessária devido à relevância científica demonstrada para a compreensão do tema. Para a realização da busca, utilizaram-se os descritores "obesity", "dislipidemy", "hypertension", "metabolic syndrome”, "exercise", "resistance training" $e$ "weight lifting”. Foram encontrados 6.311 artigos. Foram selecionados, principalmente, artigos com experimentos randomizados, controlados e que apresentaram suas respectivas análises estáticas adequadas à explicação dos objetivos propostos por cada artigo. Foram excluídos os artigos que apresentaram falhas metodológicas que limitavam a interpretação os dados.

\section{Resultados e Discussão}

\section{Associação entre força muscular e síndrome metabólica}

Jurca et al. (2004) ${ }^{4}$ realizaram um estu- 
do no qual um dos objetivos principais era examinar a associação entre a força muscular e a prevalência de síndrome metabólica. Participaram do estudo 8.570 homens, com idade de 20-75 anos. Concluiuse que a força muscular é independentemente associada à prevalência de síndrome metabólica. Os homens com maiores níveis de força tiveram uma probabilidade $67 \%$ menor de ter síndrome metabólica, comparados aos homens com menores níveis de força.

Considerando a colocação acima, é possível sugerir que o desenvolvimento da força muscular deve ser incluído nas recomendações de atividades físicas para a prevenção dos grupos de fatores de risco que caracterizam a síndrome metabólica. A seguir, será feita uma análise dos efeitos do treinamento de força sobre cada fator que caracteriza a síndrome de resistência insulínica.

\section{Treinamento de força e obesidade}

A obesidade tem sido classificada primariamente como uma desordem conseqüente de uma alta ingestão calórica ${ }^{2}$. Ela é proveniente de um desequilíbrio entre a alta ingestão e baixo gasto energético, resultando em um balanço energético positivo $^{2}$. A obesidade pode ser classificada como Índice de Massa Corporal (IMC) ${ }^{3} 30$ $\mathrm{Kg} / \mathrm{m}^{2}$, sendo definida funcionalmente como o percentual de gordura corporal no qual aumenta o risco de doenças ${ }^{9}$. O exercício faz aumentar o dispêndio calórico e torna mais lento o ritmo de perda de massa magra que ocorre quando alguém perde peso por acentuada restrição calórica ${ }^{9}$. Os mecanismos através dos quais a força muscular contribui para a diminuição da obesidade e de seus fatores de risco incluem a redução na gordura abdominal, melhoria da concentração de triglicérides no plasma, aumento do HDL-C (high density lipoprotein- colesterol) e controle glicêmico ${ }^{4}$. A partir disso, será feita uma relação entre os efeitos do treinamento de força sobre o gasto energético total, adiposidade intra-abdominal, lipase lipoprotéica e sobre a imunidade de indivíduos obesos.

\section{Treinamento de Força e Gasto Energético} Total

O gasto energético total (GET) é composto de três componentes: metabolismo de repouso (MR), termogênese induzida pela dieta (TID) e atividade física (AF). $\mathrm{O}$ MR é afetado pelo sexo, idade, estado nutricional e endócrino, e pela composição corporal. A atividade física é o componente mais variável do GET, podendo ser aumentada em dez vezes em relação à taxa metabólica de repouso ${ }^{10}$.

O TF é capaz de promover modificações agudas e crônicas no GET. As modificações agudas são aquelas do próprio custo energético para a realização de atividade e na fase de recuperação. Os efeitos crônicos são proporcionados por alterações na taxa metabólica de repouso (TMR). O fator altamente responsável pela modificação da TMR é o ganho de massa magra (MM). Para mensurar o GET durante o TF, a maior dificuldade se encontra na padronização dos estudos em relação à intensidade e ao volume utilizado. Além disso, é desconsiderado o momento pós-exercício, dificultando posterior comparação de da$\operatorname{dos}^{11}$.

Segundo Thornton et al. (2002) ${ }^{12}$, durante este tipo de exercício gasta-se de 64 a $534 \mathrm{Kcal}$, e parece que o volume do programa de treinamento é a variável mais determinante desse processo. No período de recuperação, a intensidade é fator de maior importância. De acordo com Binzen et al. (2001) ${ }^{13}$ e Thornton et al. (2002) ${ }^{12}$, no exercício contra-resistência o gasto energético representado pelo EPOC (excess post exercise oxygen consumption) varia de 6 a 114 Kcal, durando de 1 a 15 horas após o término da atividade.

Segundo Melby et al. $(1998)^{14}$, a atividade contra-resistência pode causar maior impacto sobre o EPOC durante o período de recuperação devido a um componente curto relacionado com a restaura- 
ção dos estoques de ATP e fosfocreatina muscular, ao restabelecimento do estoque de oxigênio sangüíneo e muscular, aos danos teciduais, ao aumento da FC e temperatura, à remoção de lactato e à alta atividade do sistema nervoso simpático. O componente longo está relacionado à magnitude de ativação do metabolismo anaeróbico durante o exercício e à liberação de hormônio do crescimento e cortisona. Burleson et al. ${ }^{15}$, realizaram um estudo para verificar por quanto tempo o EPOC se prolongava após uma atividade aeróbica e outra anaeróbica (TF) a $44 \%$ do $\mathrm{VO}_{2}$ por 27 minutos. Após a atividade aeróbica, o EPOC durou cerca de 30 minutos e, após a atividade anaeróbica, em torno de 90 minutos.

O mecanismo de ação do TF, por meio do EPOC, na perda de peso corporal, encontra-se no princípio da atividade de alta intensidade, na qual há maior ativação do sistema nervoso simpático, aumentando, assim, o metabolismo lipídico de repouso, mudando o substrato energético, que durante o exercício é glicogênio ${ }^{12}$.

Para o controle da obesidade, a variável mais importante a ser considerada é a intensidade, visto sua importância na oxidação lipídica em repouso, o que resultará em melhor composição corporal, através de uma melhor relação entre a massa magra e a massa adiposa ${ }^{14,16}$. Paradoxalmente, o $\mathrm{ACMS}^{6}$ preconiza que seja aplicado maior volume de treinamento. Para isso é necessário maior número de pesquisas para estabelecer qual variável do treinamento deve ser prioritariamente enfatizada.

O TF também exerce efeito sobre a TID como mostra o estudo de Denzer \& Young $(2003)^{17}$, no qual uma única sessão de TF (2 séries de 10 repetições de 10 exercícios com 40 segundos de intervalo entre as séries) foi capaz de aumentar a TID em $73 \%$ no grupo exercitado, em relação ao controle. Embora seja pequena a contribuição da TID (33 Kj/ h) para o GET, esta deve ser considerada no controle ponderal, pois, quando combinada com os outros benefícios do TF, como o aumento da massa magra e a energia gasta pela própria execução da atividade, contribui para aumentar o GET, otimizando a perda de peso corporal.

Treinamento de Força e Adiposidade Intra -abdominal

O acúmulo de gordura corporal pode ser o fator mais importante para predizer o estado de saúde do indivíduo do que propriamente a gordura total ${ }^{18}$. Está bem estabelecido que o acúmulo de gordura no tronco, e especialmente no tecido adiposo intra-abdominal (TAIA), está correlacionado com o desenvolvimento de diabetes mellitus e doenças cardiovasculares, bem como com a mortalidade ${ }^{19}$.

Hunter et al. (2004) ${ }^{1}$, em um estudo com idosos acima de 60 anos, com duração de 25 semanas, verificou que o TF (10 exercícios e duas séries de 10 repetições a $65-80 \%$ de 1 RM) foi capaz de promover uma perda de peso corporal correspondente a 1,7 kg nas mulheres e 1,8 kg nos homens. Além disso, as mulheres foram capazes de ganhar $1 \mathrm{~kg}$ de massa magra e os homens, 2,8 kg. O TAIA das mulheres diminuiu $16 \mathrm{~cm}^{2}$ e o dos homens aumentou $9 \mathrm{~cm}^{2}$, e o subcutâneo feminino diminuiu $15 \mathrm{~cm}^{2}$ e o masculino não teve alteração. Os resultados sugerem que há diferença entre os gêneros para a perda de TAIA induzido pelo TF.

Treuth et al. (1998) ${ }^{20}$ realizaram um estudo para determinar os efeitos do TF no acúmulo de gordura corporal em garotas pré-púberes obesas $(N=20)$. Iniciou-se o programa de treinamento com uma carga de $50 \%$ de $1 \mathrm{RM}$, progredindo até uma execução confortável. A adiposidade abdominal subcutânea aumentou no grupo exercitado (192 para $208 \mathrm{~cm}^{2}$ ), contudo a adiposidade intra-abdominal não mostrou diferenças significativas. Conclui-se que, para garotas em fase de crescimento, o TF, com a metodologia apresentada neste estudo, não surtiu efeitos positivos para a diminuição da gordura intra-abdominal e subcutânea. 
Treinamento de Força e os níveis de Leptina Circulante

A leptina é uma proteína secretada pelos adipócitos com papel regulador em vários sistemas do organismo, como sistema imune, respiratório e reprodutivo, bem como no balanço energético via ação hipotalâmica. Sua ação primária ocorre no núcleo hipotalâmico arqueado, no qual inicia uma cascata de eventos para inibição da ingestão energética e aumento do gasto energético. As concentrações de leptina são influenciadas pela adiposidade, fatores hormonais e nutricionais ${ }^{21}$. Sua concentração é diretamente proporcional à da massa adiposa, e mudanças nesta provenientes da perda de peso estão associadas ao correspondente declínio da leptina sistêmica ${ }^{22}$.

Nindl et al. (2002) ${ }^{22}$ realizaram um estudo com o objetivo de verificar se a concentração de leptina circulante diminuía após a sessão de TF. O estudo foi realizado com 10 homens com idade média de $21 \pm$ 1 anos e percentual de gordura de $11 \%$. $\mathrm{O}$ protocolo de exercícios consistia em 50 séries (agachamento, supino, leg press e puxador) com 10 e $5 \mathrm{RM}$ alternadas e intensidade de 70 a $85 \%$ de $1 \mathrm{RM}$, respectivamente. $\mathrm{O}$ intervalo entre as séries era de 90 segundos, tendo o programa de treinamento duração média de 123 minutos. Os resultados mostraram que, na primeira hora após o término da atividade, a leptina encontrava-se com 2,22 vs 2,67 ng.ml e após 13 horas, 2,19 vs 265 ng.ml, exercício e controle, respectivamente. Diante dos dados apresentados, conclui-se que as células gordurosas são produtoras de hormônio e sofrem influência do TF. Parece que a energia gasta durante a atividade e no EPOC prolonga os benefícios do TF, o que induz uma quebra de homeostase energética, provocando a diminuição da leptina circulante $^{22}$.

Treinamento de Força e a Atividade das Células Natural Killer

A Célula Natural Killer (NK) é um tipo de linfócito que tem importante papel no sistema imunológico contra neoplasmas e células de infecção viral. Connie et al. (1998) ${ }^{23}$ realizaram um estudo que pretendeu avaliar o efeito de oito semanas de um programa de treinamento de força combinado com treinamento aeróbico destinado à perda de peso sobre a atividade das células NK em mulheres obesas $(N=25)$, divididas em dois grupos: 12 participaram de um tratamento que consistia somente de dieta (D) e 10 de outro grupo que consistia de dieta e exercício (D+E). O exercício aeróbico consistia na realização de 12 minutos de caminhada em esteira a $40 \%$ e mais oito minutos a $50 \%$ do $\mathrm{VO}_{2}$ máximo. O TF era composto de uma série de 12 repetições a $40 \%$ de $1 \mathrm{RM}$, progredindo para 2 séries de 12 repetições a $60 \%$ do $1 \mathrm{RM}$. Os resultados evidenciam diminuição de $50 \%$ da NK (CD56+) no grupo D, mas sem alteração no grupo D+E e até aumento em 7 indivíduos deste grupo. Essa diferenciação na atividade das células NK nos dois grupos sugere que o exercício moderado foi efetivo para limitar a supressão da função das células NK provocada pela perda de peso por meio da dieta.

\section{Treinamento de força e diabetes melittus}

O diabetes mellitus é um grupo de doenças metabólicas caracterizadas por hiperglicemia, que resulta em deficiência na secreção de insulina, na ação da insulina ou ambas. A dieta é o ponto central no controle de peso e no diabetes mellitus tipo $2^{24}$. Contudo, muitas vezes a diminuição do peso pela dieta se deve à diminuição da massa magra, o que pode conseqüentemente reduzir a taxa de metabolismo basal ${ }^{24,25}$.

Uma estratégia relativamente nova para o combate da resistência insulínica é o Treinamento de Força Progressivo (TFP), que é definido como um aumento progressivo da força exercida contra uma resistência, preferido quando o paciente diabético sofre de ulcerações nos pés ${ }^{25}$. Fraqueza muscular, diminuição da massa magra, 
diminuição da atividade da enzima glicogênio sintetase e mudanças no tipo de fibra do tipo 2 para tipol são relatadas preceder a resistência insulínica, intolerância a glicose e $\mathrm{DM}^{26}$. O TF pode agir sobre essas variáveis, amenizando os sintomas negativos provocados pela DM2.

O ACSM ${ }^{24}$ recomenda o TFP duas vezes por semana, 8-10 exercícios envolvendo grupamentos musculares grandes, de no mínimo uma série de 10-15 repetições perto da fadiga. $\mathrm{A} \mathrm{ADA}^{26}$ preconiza que sejam utilizadas cargas leves e alto número de repetições para o aumento da força em todos os pacientes com DM2. O TF com intensidade entre 60 e $100 \%$ de 1 RM solicita mudanças estruturais funcionais e metabólicas nos músculos, e maiores intensidades provocam maiores adaptações ${ }^{31}$. TFP tem mostrado melhorar a taxa de eliminação de glicose, aumentar a capacidade de estoque de glicogênio, aumentar receptores GLUT 4 no músculo, aumentar a sensibilidade à insulina e normalizar a tolerância à glicose $\mathrm{e}^{28}$.

Treinamento de Força e Complicações do Diabetes Mellitus

Para diabéticos com complicações vasculares periféricas e nefropatia, o TFP apresenta grandes vantagens, pois a variedade de máquinas permite variação do trabalho muscular. Isto diminui o risco de lesões nos pés por movimentos repetitivos como ocorre durante a caminhada e outras atividades aeróbicas ${ }^{29}$.

$\mathrm{O}$ aumento da pressão sanguínea com o TFP implica cuidados com os pacientes com nefropatia, mas não há evidência de que mudança na pressão sangüínea induzida pelo exercício aumente a progressão da doença ${ }^{25}$. Contudo, é aconselhável que se evite atividades que excedam $200 \mathrm{mmHg}$ da pressão arterial sistólica, visto que uma elevação acima deste valor pode piorar a progressão da doença ${ }^{25}$. Deve-se evitar manobra de valsalva e contrações isométricas, para não aumentar a pressão intraocular $^{30}$.
Treinamento de Força, Sensibilidade a Insulina e Homeostase da Glicose Sanguínea

A perda da sensibilidade à insulina, que ocorre com a idade, se deve à diminuição da atividade física e ao aumento da obesidade central. Os exercícios físicos resultam, preferencialmente, em perda de gordura dessa região central, e parece que a perda de gordura visceral está intimamente correlacionada com o aumento da sensibilidade à insulina ${ }^{31}$.

Ibanez et al. (2005) ${ }^{32}$ avaliaram a influência do TFP na gordura abdominal e na sensibilidade à insulina de nove homens com DM2, com idade média de $66 \pm 3$ anos. O programa de treinamento foi realizado duas vezes por semana, durante 16 semanas, em uma intensidade que variou de 50 a $80 \%$ de $1 \mathrm{RM}$, sem uma dieta para perda de peso. Após o tratamento, a gordura visceral e a subcutânea tiveram diminuição significativa: de 10,3 e 11,2\%, respectivamente. O TFP aumentou a sensibilidade à insulina em 46,3\%, a glicose de jejum diminuiu 7,1\%. Com isso, concluiu-se que duas sessões de treino duas vezes por semana, sem uma dieta para a perda de peso simultaneamente, podem ser efetivas para melhorar a sensibilidade à insulina e a glicemia de jejum e diminuir a gordura abdominal em homens idosos com DM2.

O potencial mecanismo que explica como o TFP pode aumentar a sensibilidade à insulina não tem sido bem documentado. Contudo, existem evidências de que o próprio exercício promove mudanças no peso ou na composição corporal, o que é capaz de aumentar a sensibilidade à insulina significantemente ou melhorar a homeostase da glicose $\mathrm{e}^{30}$.

Dunstan et al. (2002) ${ }^{33}$ verificaram os efeitos do TFP de alta intensidade, combinado com moderada perda de peso no controle glicêmico e na composição corporal por seis meses, em 36 homens sedentários com idade entre 60 e 80 anos. O programa de treinamento era realizado três vezes por semana, durante 45 minutos. Era composto por nove exercícios, sendo executadas 3 
séries de 8-10 repetições, com intervalo de 90 a 120 segundos entre elas. Verificou-se, ao fim do tratamento, que os indivíduos treinados apresentaram diminuição de 1,2 $\pm 1 \%$ na hemoglobina glicosilada, $1,4 \pm 2,7$ $\mathrm{mmol} / \mathrm{l}$ na glicose plasmática de jejum,e aumento de $10,5 \pm 46,3 \mathrm{pmol} / 1$ nos níveis de insulina de jejum. A partir das evidências científicas apresentadas, é possível afirmar a efetividade do TF sobre os parâmetros metabólicos, podendo ser um importante componente no controle e na prevenção do DM tipo 2.

\section{Treinamento de força e dislipidemia}

A dislipidemia é caracterizada por um distúrbio no metabolismo lipídico com repercussão nos níveis de lipoproteínas na circulação sangüínea, bem como nas concentrações de seus componentes ${ }^{34}$. Níveis anormais de colesterol total, HDL-C triglicérides, lipoproteína (a) plasmática estão diretamente associados à gênese e evolução da aterosclerose ${ }^{34}$. Na dislipidemia, o LDL-C (low density lipoproteincolesterol) geralmente não se eleva significantemente, porém aumenta o número de partículas menores e densas denominadas quilomícrons $^{35 .}$

Probhakaran et al. (1999) ${ }^{36}$ realizaram um estudo com mulheres na pré-menopausa $(N=24)$, durante 14 semanas, em sessão de treinamento com 45-50 minutos de duração a $85 \%$ de 1 repetição máxima (1 RM). Os resultados constataram diminuição de $14 \%$ do LDL-C, melhorando a relação LDL-C / HDL-C. Não houve nenhuma mudança no perfil lipoprotéico do grupo controle.

Elliot et al. (2002) ${ }^{37}$ estudaram os efeitos de 8 semanas de TF de baixa intensidade no perfil lipídico de 15 mulheres sedentárias na pós-menopausa. O programa de treinamento consistia de três séries, com oito repetições com intensidade equivalente a $80 \%$ de $10 \mathrm{RM}$. Os resultados mostraram que não houve mudanças significativas no perfil lipídico após o TF.

\section{Treinamento de força e hipertensão arterial}

Restrição de sal, consumo moderado de álcool, redução de peso e aumento da atividade física são recomendações para o controle e tratamento da hipertensão arterial (HA) ${ }^{38}$. O objetivo da prevenção e controle da hipertensão arterial consiste em reduzir a morbidez e a mortalidade pelo meio menos invasivo possível. Isso é possível, mantendo-se uma pressão arterial sistólica menor que $140 \mathrm{mmHg}$ e diastólica menor 90 mmHg; para isso, são necessárias modificações no estilo de vida $^{6}$.

As V Diretrizes Brasileiras de Hipertensão (2006) ${ }^{39}$ recomendam a prática regular de exercícios físicos para todos os hipertensos, inclusive para aqueles sob tratamento medicamentoso, porque reduz a pressão arterial sistólica/diastólica em 6,9/ 4,9 mmHg. Estabelece que esse grupo deva treinar de 3-5 vezes por semana por pelo menos 30 minutos e, com o objetivo de emagrecimento, por 60 minutos. Preconiza que, além do treinamento aeróbico, os hipertensos devem praticar exercícios resistivos. A intensidade do treino deve ser de $50-60 \%$ de 1 RM e o exercício deve ser interrompido quando a velocidade de movimento diminuir (antes da fadiga concêntrica).

O Colégio Americano de Medicina do Esporte $^{9}$ recomenda que o TF deve ser executado com baixa resistência e alto número de repetições. Uma das recomendações mais importantes que o ACSM faz é de que a Manobra de Valsalva (MV) deve ser evitada, em razão de potencial risco de acidente vascular cerebral devido ao aumento da pressão arterial. Contudo, Haykowsky et al. $(2003)^{40}$ examinaram os efeitos agudos do treinamento de força realizado sem MV e a realização da MV sozinha sobre a pressão sistólica (PS), a pressão intracraniana (PI) e a pressão transmural cérebro vascular (PT = PS $-\mathrm{PI}$ ), sendo esta responsável pelos aneurismas, e puderam verificar que durante o exercício a PT é maior, porém a PI tam- 
Tabela 1 - Efeito do Treinamento de força sobre os fatores de risco da síndrome metabólica. Table 1 - Effects of resistance training on metabolic syndrome risk factors

\begin{tabular}{|c|c|c|c|c|}
\hline $\begin{array}{l}\text { Denzer \& Young } \\
(2003)^{15}\end{array}$ & $\begin{array}{l}\text { Efeito do TF sobre a TID. } \\
\text { ( } \mathrm{n}=9 ; \text { ambos os sexos; } \\
\text { idade }=22 \pm 1 \text { anos) }\end{array}$ & $\begin{array}{l}\text { Única sessão de treinamento } \\
\text { Séries: } 2 \\
\text { Repetições: } 10 \\
N^{0} \text { exercícios: } 10 \text { Intervalo } \\
\text { entre as séries: } 40 \text { segundos }\end{array}$ & $\begin{array}{l}\text { Após TF a TID aumentou } \\
73 \% \text { em relação ao } \\
\text { grupo controle }\end{array}$ & $\begin{array}{l}\text { TF é importante para o } \\
\text { controle ponderal. }\end{array}$ \\
\hline $\begin{array}{l}\text { Hunter et al. } \\
(2004)^{16}\end{array}$ & $\begin{array}{l}\text { Efeito do TF sobre TAIA. } \\
(\mathrm{n}=30 ; \text { ambos os sexo; } \\
\text { idade }=65 \pm 9 \text { anos) }\end{array}$ & $\begin{array}{l}\text { Duração: } 25 \text { semanas } \\
\text { Séries: } 2 \\
\text { Repetições: } 10 \\
\mathrm{~N}^{0} \text { exercícios: } 10 \\
\text { Intensidade: } 65-80 \% \text { de } 1 \text { RM }\end{array}$ & $\begin{array}{l}\text { Com oTF, o TAIA das } \\
\text { mulheres diminuiu (de } \\
131 \text { para } 116 \mathrm{~cm}^{2} \text { ) e dos } \\
\text { homens aumentou (de } \\
143 \text { para } 152 \mathrm{~cm}^{2} \text { ) }\end{array}$ & $\begin{array}{l}\text { Provável diferença } \\
\text { entre os gêneros para a } \\
\text { perda de TAIA induzida } \\
\text { por TF. }\end{array}$ \\
\hline $\begin{array}{l}\text { Treuth et al. } \\
(1998)^{18}\end{array}$ & $\begin{array}{l}\text { Efeito do TF no acúmulo de } \\
\text { gordura corporal. } \\
\text { ( } \mathrm{n}=20 \text {; garotas de } 7 \text { - } 10 \\
\text { anos). }\end{array}$ & $\begin{array}{l}\text { Duração: } 5 \text { meses } \\
\text { Freqüência semanal: } 3 \\
\text { Duração da sessão: } 20 \text { minutos } \\
\text { Séries: } 2 \\
\text { Repetições: } 12 \text { (MS) e } 15 \text { (MS) } \\
\text { Noexercícios: } 7 \\
\text { Intensidade: } 50 \text { \% de } 1 \text { RM }\end{array}$ & $\begin{array}{l}\text { T AS aumentou no grupo } \\
\text { exercitado (192 para } 208 \\
\mathrm{~cm}^{2} \text { ), contudo a TAIA } \\
\text { não mostrou diferenças } \\
\text { significativas. }\end{array}$ & $\begin{array}{l}\text { OTF, com a } \\
\text { metodologia } \\
\text { apresentada, não surtiu } \\
\text { efeitos positivos para a } \\
\text { diminuição de TAS e } \\
\text { TAIA. }\end{array}$ \\
\hline $\begin{array}{l}\text { Connie et al. } \\
(1998)^{24}\end{array}$ & $\begin{array}{l}\text { Avaliar o efeito de TF }+ \text { TA } \\
\text { sobre a atividade das células } \\
\text { NK. }(n=25 \text { mulheres obesas; } \\
\text { idade }=37 \pm 6 \text { anos })\end{array}$ & $\begin{array}{l}\text { Duração do estudo: } 8 \text { semanas } \\
\text { Grupo D e Grupo D+E } \\
\text { Série: } 1 \\
\text { Repetições: } 12 \\
\text { Intensidade: Inicialmente } 40 \% \\
\text { de } 1 \text { RM, progredindo para } 2 \\
\text { séries de } 12 \text { repetições a } 60 \% \\
\text { do } 1 \text { RM }\end{array}$ & $\begin{array}{l}\text { Diminuição de } 50 \% \text { da } \\
\text { NK (CD56+) no grupo D, } \\
\text { mas sem alteração no } \\
\text { grupo D+E. }\end{array}$ & $\begin{array}{l}\text { Exercício moderado foi } \\
\text { efetivo para limitar a } \\
\text { supressão da função } \\
\text { das células NK } \\
\text { aumentando, assim, a } \\
\text { função imune. }\end{array}$ \\
\hline $\begin{array}{l}\text { Ibanez et al. } \\
(2005)^{29}\end{array}$ & $\begin{array}{l}\text { Influência do TFP na gordura } \\
\text { abdominal e na sensibilidade } \\
\text { à insulina. } \\
\text { ( } \mathrm{n}=9 \text { homens com DM } 2 \text {; } \\
\text { idade }=66 \pm 3 \text { anos) }\end{array}$ & $\begin{array}{l}\text { Duração do estudo: } 16 \text { semanas } \\
\text { Freqüência semanal: } 2 \\
\text { Primeiras } 8 \text { semanas: } \\
\text { Intensidade: } 50 \text { a } 80 \% \text { (nas } \\
\text { oito primeiras semanas, } \\
\text { intensidade de } 50 \text { a } 70 \% \text { e } 3 \text { a } \\
4 \text { séries de } 10-15 \text { repetições; e } \\
\text { nas últimas oito semanas, } \\
\text { intensidade de } 70 \text { a } 80 \% \text { e } 3 \text { a } \\
5 \text { séries de } 5-6 \text { repetições de } \\
1 \text { RM) }\end{array}$ & $\begin{array}{l}\text { GV e GS tiveram } \\
\text { diminuição significativa: } \\
\text { de } 10,3 \text { e } 11,2 \% \text {, } \\
\text { respectivamente. O TFP } \\
\text { aumentou a } \\
\text { sensibilidade à insulina } \\
\text { em } 46,3 \% \text {. }\end{array}$ & $\begin{array}{l}\text { TF sem uma dieta para } \\
\text { a perda de peso } \\
\text { simultaneamente, pode } \\
\text { ser benéfico para } \\
\text { homens idosos com } \\
\text { DM2. }\end{array}$ \\
\hline $\begin{array}{l}\text { Dunstan et al. } \\
(2002)^{31}\end{array}$ & $\begin{array}{l}\text { Efeitos do TFP de alta } \\
\text { intensidade + moderada } \\
\text { perda de peso no controle } \\
\text { glicêmico e na composição } \\
\text { corporal. } \\
\text { ( } \mathrm{n}=36 \text { homens sedentários; } \\
\text { idade }=60-80 \text { anos) }\end{array}$ & $\begin{array}{l}\text { Freqüência semanal: } 3 \\
\text { Duração sessão: } 45 \text { minutos } \\
N^{0} \text { de exercícios: } 9 \\
\text { Séries: } 3 \\
\text { Repetições: 8-10 } \\
\text { Intervalo de } 90 \text { a } 120 \\
\text { segundos }\end{array}$ & $\begin{array}{l}\text { Diminuição: } \mathrm{Hb}_{\text {glic }} 1,2 \pm \\
1 \% \text { e } 1,4 \pm 2,7 \mathrm{mmol} / \mathrm{l} \mathrm{na} \\
\text { GPJ. Aumento de } 10,5 \pm \\
46,3 \text { pmol/l nos níveis de } \\
\text { insulina de jejum. }\end{array}$ & $\begin{array}{l}\text { TF pode ser um } \\
\text { importante } \\
\text { componente no } \\
\text { controle e na } \\
\text { prevenção do DM2. }\end{array}$ \\
\hline $\begin{array}{l}\text { Elliot et al. } \\
(2002)^{34}\end{array}$ & $\begin{array}{l}\text { Efeito do TF no perfil lipídico. } \\
\text { ( } \mathrm{n}=15 \text { mulheres sedentárias; } \\
\text { idade }=49 \text { a } 62 \text { anos). }\end{array}$ & $\begin{array}{l}\text { Duração do estudo: } 8 \text { semanas } \\
\text { Séries: } 3 \\
\text { Repetições: } 8 \\
\text { Intensidade: } 80 \% \text { de } 10 \mathrm{RM}\end{array}$ & $\begin{array}{l}\text { Não houve mudanças } \\
\text { significativas no perfil } \\
\text { lipídico após o TF. }\end{array}$ & $\begin{array}{l}\text { O TF não foi efetivo } \\
\text { para uma melhoria do } \\
\text { perfil lipídico desta } \\
\text { população especifica. }\end{array}$ \\
\hline
\end{tabular}


bém tem maior elevação quando se comparam os valores de MV executada sozinha (31 \pm 14 e $16 \pm 7$ mmHg, respectivamente), o que acarreta menor pressão transmural cerebrovascular $(132 \pm 14 \mathrm{mmHg}$ em exercício sem MV e $106 \pm 22 \mathrm{mmHg}$, somente MV). Segundo esses autores, a Manobra de Valsalva poderia diminuir o estresse cerebral em $11 \%$, podendo ser um elemento protetor para o sistema cérebro-vascular. Contudo, a aplicação de MV para hipertensos não é recomendada, pois faltam evidências cientificas que garantam a segurança e a eficácia da execução de MV durante a realização de exercícios resistivos.

\section{O TF e a Liberação de Vasodilatadores Durante o Exercício}

Uma variedade de substâncias vasodilatadoras parecem ser liberadas na microvascularização durante o exercício físico, como a prostaciclina $\left(\mathrm{PGI}_{2}\right)$, a adenosina $(\mathrm{AD})$, sendo o óxido nítrico (NO) o mais significativo. Além de sua função vasodilatadora está relacionado com a expressão e regulação do gene que produz fator de crescimento angiogênico no endotélio vascular. A hipótese para a secreção de NO após o exercício vigoroso baseia-se no fato de que o estresse provocado estimula o endotélio microvascularizado a secretar substâncias como um sinal da necessidade de aumentar a vascularização, a fim de melhorar a capacidade das células em estar executando exercícios vigorosos ${ }^{41}$.

Benoit et al. (1999) ${ }^{41}$ realizaram um estudo que pretendia verificar se substâncias vasodilatadoras aplicadas localmente por infusão tinham capacidade de potencializar a expressão do gene de fator de crescimento angiogênico no músculo em repouso. A amostra era constituída de 7 grupos (grupo-controle com infusão salina no músculo gastrocnêmio e grupos-teste com as seguintes infusões: Nitroprusside (NP), Acetilcolina (Act), Prostaglandinas $\left(\mathrm{PGE}_{1} \mathrm{e}\right.$ $\left.\mathrm{PGE}_{2}\right)$, Prostaciclina $\left(\mathrm{PGI}_{2}\right)$ e Adenosina (AD), com 5 a 7 ratos Wistars em cada. NO, $\mathrm{AD}$ e $\mathrm{PGI}_{2}$ foram liberadas durante a prática de atividade física. Os resultados mos- traram que a Act e NP, doadores de NO, aumentaram em $50 \%$ o fator de crescimento vascular endotelial (FCVE), e que $\mathrm{PGI}_{2}$ diminuiu em 40\% FCVE; $\mathrm{PGE}_{1}, \mathrm{PGE}_{2}$ e AD não alteraram a concentração de FCVE.

Com o avanço da idade, o fluxo sangüíneo mediado pela vasodilatação é prejudicado, principalmente nas arteríolas dos músculos em que predominam fibras de contração rápida ${ }^{42}$. O TF, executado com cargas de média a alta intensidade, excita, prioritariamente, as fibras de contração rápida, podendo ser um componente valioso para a formação de microcirculação nesta musculatura. Concluiu-se que os principais reguladores da expressão gênica do FCVE são o NO, que aumenta a expressão, e o $\mathrm{PGI}_{2}$ que atua negativamente.

\section{Comentários finais}

Esta revisão de literatura propõe que o aumento da força muscular parece ter efeito protetor na prevalência da síndrome metabólica. Desta forma, a prática de exercícios resistidos parece agir positivamente sobre o controle dos fatores de risco dessa enfermidade. O TF age na perda de peso corporal pelo fato de aumentar o gasto energético total e o EPOC, aumenta a termogênese induzida pelo alimento e a atividade da leptina, contribui para o ganho de massa corporal magra e diminui o tecido adiposo visceral (o maior determinante da síndrome). Além disso, o TF é responsável por suprimir a função das células NK, aumentando, assim, a função imune, sendo importante fator no processo de angiogênese. Alguns estudos alegam contribuir para a melhoria da homeostase da glicose sangüínea, aumentando a sensibilidade dos receptores celulares à insulina podendo dessa forma exercer um papel fundamental no controle do diabetes mellitus. O TF parece agir positivamente sobre o perfil lipídico, uma vez que alguns estudos mostram uma diminuição do LDL-C, melhorando a relação LDL-C/HDL-C. O TF também é responsável por reduzir a pressão arterial sistólica/diastólica, promover a 
secreção de substancias vasodilatoras e, além disso, parece ser um componente valioso para a formação de microcirculação na musculatura exercitada.

Considerando os estudos apresentados, o treinamento de força pode ser considerado um componente indispensável em um programa de aptidão física bem elaborado, que objetive a prevenção de um estado saudável, ou mesmo a reversão ou otimização dos fatores de risco já instalados nos pacientes com síndrome metabólica. O resumo dos estudos apresentados na presente revisão de literatura estão expostos na Tabela 1 .

\section{Referências}

1. Word Health Organization. Obesity: preventing managing the global epidemic. Geneva: Word Health Organization; 1998.

2. Ciolac GM, Guimarães GV. Exercício físico e síndrome metabólica. Rev Bras Med Esporte 2004; 10(4): 319-23.

3. Gurruchaga AM. Consecuencias Patologicas de la Obesidad: Hipertension Arterial, Diabetes Mellitus y Dislipidemia. Boletín Escuela de Medicina. Pontificia Universidad Católica de Chile 1997; 26: 18-21. Disponível em http://escuela.med.puc.cl/publicaciones/ boletin/ html/obesidad/obesidad05.html.

4. Jurca R, Lçamonte MJ, Church ST, Earnest CP, Fitzgerald SJ, Barlow CE et al. Association of muscle strength and aerobic fitness with metabolic syndrome in men. Med Sci Sports Exerc 2004; 36(8) : 1301-7.

5. Pate RR, Pratt M, Blair SN, Haskell WL, Macera CA, Bouchard C, et al. Physical activity and public health: A recommendation from the centers for disease control and prevention and the American College of Sports Medicine.[Special Communication]. JAMA 1995; 273: 4027 .

6. American College of Sport Medicine. Position Stand: The recommended quantity and quality of exercise for developing and maintaining cardiorespiratory and muscular fitness, and flexibility in health adults. Med Sci Sports Exerc 1998; 30: 975-91.

7. American College Sports Medicine. Progression models in resistance training for healthy adults. Med Sci Sports Exerc 2002; 34(2): 364-80.

8. Kraemer WJ, Ratamess NA, French, DN. Resistance Training for Health and Performance). Curr Sports Med Rep 2002; 1: 165-71.

9. American College Sports Medicine. Diretrizes do ACMS para os testes de esforço e sua prescrição. $6^{\mathrm{a}} \mathrm{Ed}$. Guanabara Koogan; 2003. Cap.10. p.136-41.

10. Kraemer WJ, Volek JS, Clark KL, Gordon SE, Incledon T, Puhl SM, et al. Physiological adaptations to a weight-loss dietary regimen and exercise programs in women. J Appl Physiol 1997; 83: 270-9.
11. Meirelles CM, Gomes SC. Efeitos agudos da atividade contra-resistência sobre o gasto energético: revisando o impacto sobre as principais variáveis. Rev Bras Med Esporte 2004; 10(2): 122-30.

12. Thornton K, Potteiger JA. Effects of resistance exercise bouts of different intensities but equal work on EPOC. Med Sci Sports Exerc 2002; 34: 715 -22.

13. Binzen CA, Swan PD, Manore M. Excess Post exercise oxygen consumption and substrate use after resistance exercise in Women. Med Sci Sports Exerc 2001; 33:32-8.

14. Melby CL, Commerford SR, Hill JO. Exercise, macronutrient balance, and weight control. In: Lamb, DR, Murray R. Perspectives in exercise science and sports medicine: exercise, nutrition, and weight control. Carmel: Cooper Publication Group; 1998. p. 1-60.

15. Burleson MA, O'Bryant HS, Stone MH, Collins MA Triplet-McBride T. Effect of weight training and treadmill exercise on post exercise oxygen consumption. Med Sci Sports Exerc 1998; 30: 518-22.

16. Elliot DL, Goldberg L, Kuel KS. Effects of resistance exercise on excess post-exercise oxygen consumption. $J$ Appl Sports Sci Res 1992; 6: 77-81.

17. Denzer C.M, Young JC. The effects of resistance exercise on the thermic effect food. Int J Sport Nutr Exerc Metab 2003; 13: 396-402.

18. Hunter GR, Bryan DR, Wetzstein CJ, Zuckerman PA, Bamman MM. Resistance training and intra- abdominal adipose tissue in older men and women. Med Sci Sports Exerc 2002; 34 (6): 1023-8.

19. Bush PA, Zuckerman AE, Taggart VS, Theiss PK, Peleg EO, Smith SA, et.al. Cardiovascular Risk in facture prevention in black school children the "Know your Body" Evaluation project. Health Educ Q 1989; 16: 215 -77.

20. Treuth MS, Hunter GR, Colon RF, Goran MI. Effects of strength training on intra- abdominal adipose tissue in obese prepubertal girls. Med Sci Sports Exerc 1998; 30(12): 1738-46.

21. Hermsdorff HHM, Vieira MAQ, Monteiro, JBR. Leptina e sua influência na patofisiologia de distúrbios alimentares. Rev Nutr 2006; 19(3): 369-79. 
22. Nindl BCW, Kraemer J, Arcieiro PJ, Samatallee N, Leone C, Mayo M, et al. Leptin concentrations experience a delayed reduction after resistance exercise in men. Med Sci Sports Exerc 2002; 34 (4): 608-13.

23. Connie BS, Verde TJ, Paolone AM, Andersen RE. Effects of Weight loss and exercise training on natural Killer cell activity in obese Women. Med Sci Sports Exerc 1998; 30(12): 1666-71.

24. American College of Sports Medicine: Appropriate intervention strategies for weight loss and prevention of regain for adults. Med Sci Sports Exerc 2001; 33: 2145-56.

25. Albright A, Franz M., Horns G, Kriska A, Marrero D, Ulrich I, et al. American College of Sports Medicine Position Stand: exercise and type 2 diabetes. Med Sci Sports Exerc 2001; 32(12): 1345-60.

26. American Diabetes Association: Diabetes Mellitus and exercise (Position Statement). Diabetes Care. 2001; 24 (Suppl. 1): S51-55.

27. Kraemer W, Deschenes M, Fleck S. Physiological adaptations to resistance exercise: implications for athletic conditioning. Sports Medicine. 1988; 6:246-56.

28. Miller J, Pratley RE, Goldberg AP, Gordon P, Rubin M, Treuth MS, Ryan AS, Hurley BF. Strength training increases insulin action in healthy 50- to 65-yr-old men. Journal Applied Physiology.1994; 77:1122-27.

29. Willey KA, Singh MAF. Battling insulin resistance in elderly obese people with type 2 diabetes. Diabetes Care 2003; 26(5): 1580-88.

30. Aiello LP, Cahill MT, Wong JS. Systemic considerations in the management of diabetic retinopathy. Am J Ophthalmol 2001; 132: 760-76.

31. Boule NG, Haddad E, Kenny GP, Wells GA, Sigal RJ. Effects of exercise on glycemic control and body mass in type 2 diabetes mellitus. JAMA 2001; 286: 1218-27.

32. Ibanez J, Mikel I, Naki AU, Luis F, Jose LL, Marisol GU, Fernando I, Esteban MG. Twice-weekly progressive resistance training decreases abdominal fat and improves insulin sensitivity in older men with type 2. Diabetes Care 2005; $28: 662-7$.
33. Dunstan DW, Daly RM, Owen N, Jolley D, Courten JS, Zimmet P. High-Intensity Resistance Training Improves Glycemic Control in Older Patients With Type 2 Diabetes. Diabetes Care 2002; 25: 1729-36.

34. Prado SE, Dantas EH.M. Efeitos dos Exercícios Físicos Aeróbio e de Força nas lipoproteínas HDL, LDL e Lipoproteína(a). Arq Bras Cardiol 2002; 79(4): 429-33.

35. Yu JN, Cunningham JA, Thhouin SR, Gurvich T, Liu D. Hiperlipidemia. Prim Care 2000; 27: 541-87.

36. Prabhakaran B, Dowling EA, Brancch JD, Swain DP, Leutholtz BC. Effect 14 week of resistance training on lipid profile and body fat percentage in premenopausal women. Br J Sports Med 1999; 33: 190-5.

37. Elliott KJ, Sale C, Cable NT. Effects of resistance training and detraining on muscle strength and blood lipid profiles in postmenopausal women. Br J Sports Med 2002; $36 ; 340-4$.

38. Fagard RH. Physical activity in the prevention and treatment of hypertension in the obese. Med Sci Sports Exerc 1999. 31(11): S624-30.

39. Sociedade Brasileira de Cardiologia, Sociedade Brasileira de Hipertensão e Sociedade Brasileira de Nefrologia. $V$ Diretrizes Brasileiras de Hipertensão Arterial. São Paulo; 2006.

40. Haykowsky MJ, Eves ND, Warburton DER, Findlay MJ. Resistance exercise, the Valsalva Maneuver and Cerobrevascular Transmural Pressure. Med Sci Sports Exerc 2003; 35(1): 65-8.

41. Benoit H, Jordan M, Wagner H, Wagner PD. .Effect of NO, vasodilator prostaglandins, and adenosine on skeletal muscle angiogenic growth factor gene expression. J Appl Physiol 1999; 86(5): 1513-8.

42. Frank AD. .Ageing, exercise training, and resistance vessels: more than just no NO ? Physiol Soc 2004;556 (3): 673.

Recebido em: 09/11/06 Versão final reapresentada em: 14/10/07 Aprovado em: 19/12/07 